\title{
Cognitive Status and Functional Dexterity as Outcome Predictors Following Urinary Diversion: A Combined Retrospective and Prospective Observational Study
}

\author{
Charis Kalogirou Svenja Breshnev Markus Krebs Lukas Koneval \\ loannis Sokolakis Hubert Kübler Hubertus Riedmiller Arkadius Kocot \\ Department of Urology and Paediatric Urology, Julius Maximilians University Medical Center of Würzburg, \\ Würzburg, Germany
}

\section{Keywords \\ Urinary diversion - Functional dexterity - Cognitive status . \\ Mini-mental test $\cdot$ Clock-drawing test $\cdot$ Medical care}

\begin{abstract}
Purpose: The study aimed to evaluate the impact of the validated functional dexterity test and the Mini-Mental Status test on subjective functional outcomes, medical care situation, and health-related quality of life (HRQoL) after urinary diversion (UD). Patients and Methods: A total of 106 patients ( $n=26$ ileal conduits, $n=29$ neobladders, and $n=51$ ileocecal pouches) were included in this combined retrospective $(n=77)$ and prospective $(n=29)$ observational study. All patients performed the 2 tests mentioned above and filled out self-designed questionnaires with diversion and HRQoL items. In the prospective cohort, the tests were performed preoperatively and the questionnaires were filled out preoperatively as well as 3 and 6 months after surgery. Results: Reduced dexterity and cognitive skills were significantly associated with increased patient age and subjective constraints in stoma care of ileal conduits, self-catheterization in ileocecal pouches, and continence in neobladders. Overall HRQoL, however, was not affected by dexterity or
\end{abstract}

๑) 2018 S. Karger AG, Basel

\section{KARGER}

E-Mail karger@karger.com

www.karger.com/uin cognitive measures. Conclusions: Assessing the cognitive status and functional dexterity of patients undergoing UD might provide a useful objective clinical tool to aid in decision-making regarding the type of UD and postoperative medical care situation. Further prospective data are needed to confirm these findings and further simplify the methods used here.

(c) 2018 S. Karger AG, Basel

\section{Background}

The history of using bowel for bladder augmentation or substitution began over 120 years ago. Tizzoni and Foggi [1] anastomosed an ileal loop with the bladder neck of dogs after cystectomy in 1888 and since this time, many techniques have been published reporting on experience in utilizing intestine for urinary diversion (UD) [2]. Besides wet loop diversions such as the ileal conduit, starting in the 1980s, a growing number of techniques for continent orthotopic or cutaneous UD have been established and described [2-4]. Long-term follow-up, for example, in the Mainz ileocecal pouch, confirmed safety and reliability of these techniques regarding complica- 
Table 1. Clinical demographics

\begin{tabular}{lccc}
\hline Variable & Combined cohort & Retrospective cohort & Prospective cohort \\
\hline No. of patients, $n$ & 106 & 77 & 29 \\
Patient age, median (range) & $66(19-90)$ & $54.5(19-90)$ & $70(42-85)$ \\
Average Karnovsky index & $90(50-100)$ & $75(50-100)$ & $80(60-100)$ \\
BMI, median (range) & $26(19.0-39.1)$ & $29.1(19.0-39.1)$ & $28.4(19.6-37.1)$ \\
HRQoL, median (range) & $76.1(36.4-94.8)$ & $77.0(36.4-94.8)$ & $67.0(41.0-92.0)$ \\
Underlying condition, $n(\%)$ & & & $5(17.2)$ \\
$\quad$ Benign & $31(29.2)$ & $26(33.8)$ & $24(82.8)$ \\
$\quad$ Malign & $75(70.8)$ & $51(66.2)$ & $12(41.4)$ \\
Previous chemotherapy, $n(\%)$ & $28(26.4)$ & $16(20.8)$ & $17(58.6)$ \\
$\quad$ Yes & $78(73.6)$ & $61(79.2)$ & $13(44.8)$ \\
$\quad$ no & $26(24.5)$ & $13(16.8)$ & $10(34.5)$ \\
Type of urinary diversion, $n(\%)$ & $29(27.3)$ & $19(24.7)$ & $6(20.7)$ \\
$\quad$ Ileal conduit & $51(48.2)$ & $45(58.5)$ & $37.3(16.0-58.5)$ \\
$\quad$ Neobladder & & $28(26-30)$ \\
$\quad$ Pouch & $28.8(16-76.1)$ & $29.5(16.5-76.1)$ & $1.5(1-2)$ \\
Performance tests & $29(11-30)$ & $20.5(11-30)$ & $2(1-3)$ \\
$\quad$ FDS score, s, median (range) & $1.73(1-3)$ & \\
$\quad$ MMS score, median (range) & & \\
Clock drawing score, median (range) & & \\
\hline
\end{tabular}

tions, renal function, metabolic consequences, body image, and health-related quality of life (HRQoL) measures [5-10].

It is well known that our society ages [11] which particularly pertains to the growing urologic patient population. Therefore, in the authors' opinion, especially the functional aspects and the medical care situation prior and after UD will have a significant impact in UD patient care in the future: As a general rule, patients should be physically and mentally able to, for example, conduct autonomous stoma care or catheterize an orthotopic or cutaneous UD. Notably, these generally accepted preconditions have never been objectively defined in the past. As the act of catheterization places higher physical and mental demands on patients than stoma care (considering the growing role of stoma therapists [12]), proper preoperative patient selection is necessary. To date, besides objective parameters such as the underlying disease or anatomical considerations, the selection and recommendation which UD to use is governed by subjective evaluation of the treating clinician.

Therefore, in this combined retrospective and prospective observational study, we sought to assess usability of 3 validated clinical tests (Functional Dexterity test [FDS] [13] and the Mini-Mental Status test [MMS]/clockdrawing test $[14,15])$ on the functional outcome after UD to potentially ease this decision in the future.

\section{Patients and Methods}

A total of 106 patients with UDs were included in the study, divided into 77 retrospectively assessed patients recruited from routine follow-up consultations (median follow-up of 71.5 months after UD) and a prospective observational cohort of 29 patients receiving cystectomy and UD at our department who were followed 6 months after surgery. UD patients contacting our department with new onset problems or late complications with regard to stoma care, self-catheterization (CIC), or continence were excluded from the study. The demographic details of the whole cohort are shown in Table 1. An ethics statement (No. 276/16) of the University of Würzburg was initiated for this study and all participating patients provided written and informed consent prior to being enrolled.

All patients were asked to perform the FDS and MMS/clock drawing tests according to the already published standardized protocols [13-16]. For the clock drawing test, we used the scoring method described by Mainland and Shulman [15]. For a brief description of the FDS test, patients were asked to turn over 16 wooden sticks on a board against the clock with their dominant hand. Dropping sticks or deviating from the tests' standardized protocol $[13,16]$ resulted in additional penalty time. Time spans ranging from 15 to $33 \mathrm{~s}$ to complete the test were regarded as sufficient dexterity, time spans over $33 \mathrm{~s}$ were regarded as reduced dexterity [16]. For the MMS test, 28 or more achieved points were regarded as adequate cognitive function. Since the MMST can be subject to a plethora of possible interference [17], this study paid special attention to reducing these factors: one single examiner conducted all MMS and FDS tests in an interferencefree environment (separate examination room) to exclude interobserver bias, and patients with illiteracy or uncorrected hearing disability were excluded from the study. After completion of the 
tests, patients were asked to fill out self-designed questionnaires (online suppl. Document 1; for all online suppl. material, see www.karger.com/doi/10.1159/000494565), assessing functional parameters, HRQoL items, and sexuality. These questionnaires were designed in the resemblance of already validated QoL assessment tools such as the S-36 questionnaire [18, 19] adding diversion-related questions for each pre-existing UD (ileal conduit, orthotopic, or cutaneous UD). Overall QoL from these selfdesigned questionnaires was then assessed using a scoring system (online suppl. Table 1). For prospectively assessed patients, in addition of the 2 tests performed, a generally designed preoperative questionnaire was handed out prior to cystectomy and creation of a UD. Three and 6 months after surgery, the patients were contacted again and asked to fill out the questionnaires for their respective UD mentioned above. The 2 tests (FDS \& MMS/ clock drawing test) were not repeated in the follow-up period in this patient group. Neither the preoperatively achieved dexterity scores nor the results of the preoperative questionnaire had an impact on the recommended and ultimately chosen UDs, according to the observational nature of this study. As setting up this study as a prospective randomized trial would have been difficult due to ethical concerns, we have conducted a combined retrospective and prospective observational study. As sampling bias was likely to occur in the prospective study cohort due to limited participating patients and 3 different types of UDs compared, the retrospective and prospective patient cohorts were combined and jointly analyzed.

For statistical evaluation, R 3.10 (https://www.r-project.org) was used. After data acquisition, Pearson's chi-square test was used to realize intergroup comparison. If 2 means of normally distributed data had to be compared, 2 -sided unpaired student's $t$ test was used. More than 2 groups were differentiated by forward analysis of variance with post-hoc testing (Tukey's test) if significant differences occurred. Significant associations were set as $p \leq 0.05\left(^{*}\right)$.

\section{Results}

General Observations Regarding the FDS and MMS

Tests and HRQoL in the Overall Patient Cohort

For the overall patient cohort, we found that reduced dexterity as well as lower MMS tests scores were significantly correlated to increased patient age in Pearson's correlation (Pearson $\rho=-0.45$ and $-0.29, p=0.02, p=$ 0.04 , respectively). Lower MMS test scores were also significantly correlated to reduced dexterity (Pearson $\rho=$ $-0.53, p=0.01$ ). Interestingly, neither reduced dexterity nor low MMS test scores were significantly correlated to poorer or improved HRQoL (n.s., Pearson $\rho=-0.13$ and 0.08 , respectively; Fig. $1 \mathrm{a}-\mathrm{c}$ ) as overall median HRQoL measures were comparable both in the prospective- and retrospective patient cohorts (74 and 77\%, respectively). It should be noted that median HRQoL measures increased significantly in the prospective cohort 6 months after surgery with rapprochement to the retrospective pa- tient cohort $(74-80 \%, p=0.04$, Fig. $1 \mathrm{~d})$. We have also tested and excluded other potential confounders regarding HRQoL: Neither the underlying condition (benign vs. malign) nor the need for revisional surgery or adjuvant chemotherapy in the postoperative period was significantly associated to poorer or better HRQoL (online suppl. Fig. 1).

\section{FDS and MMS Tests and HRQoL in Dependence of the Different Urinary Diversions}

After discriminating the overall patient cohort in $3 \mathrm{UD}$ groups (ileal conduit vs. neobladder and ileocecal pouch), we were able to demonstrate that patients receiving an ileal conduit were significantly older ( 77 vs. 66 years and 62 years, $p \leq 0.01$ ) and less dexterous than patients receiving a continent solution (median FDS 33.8 vs. $26.6 \mathrm{~s}$ and $26.7 \mathrm{~s}, p=0.01)$. For MMS test scores, patients with an ileal conduit achieved lower median test scores ( 28 vs. 29 and $29, p=0.118$ ) than patients with a continent UD. However, this difference did not reach statistical significance. For overall HRQoL, no statistical significances were found among the 3 types of UD ( 79 vs. $81 \%$ and $80 \%$, $p=1.2$ ). These results are subsumed in Fig. 2 .

\section{FDS and MMS Tests in Relation to Subjective \\ Functional Outcome after Urinary Diversion}

To assess the functional outcome of the UDs, we added items concerning stoma care, CIC, and continence in the respective questionnaires handed out to the patients (refer to item 17 in the postoperative questionnaires, online suppl. Document 1). No constraints in stoma care, $\mathrm{CIC}$, and continence were defined as complete freedom from symptoms (item weight 0 ), any or moderate constraints as item weights $1-2$, and severe constraints as item weights $>3$.

We found that reduced dexterity was significantly associated with increased constraints in stoma care, CIC, and continence (Fig. 3a): $52.6 \%$ of the patients with reduced dexterity (FDS $>33$ s) reported any or moderate constraints in comparison to $32.3 \%$ of the patients with adequate dexterity (FDS $<33 \mathrm{~s}, p \leq 0.01$ ). Moreover, severe constraints were reported in $28.9 \%$ of the patients with reduced dexterity compared to $14.7 \%$ of patients with adequate dexterity $(p \leq 0.01)$. For MMS test results, we found that patients with a reduced MMS score $(<28)$ reported significantly more moderate $(41.9$ vs. $30.1 \%, p=0.02)$ and severe constraints (23.3 vs. $14.3 \%, p=0.03$ ) concerning stoma care, CIC, and continence. A summary of these results is displayed in Figure 3. 


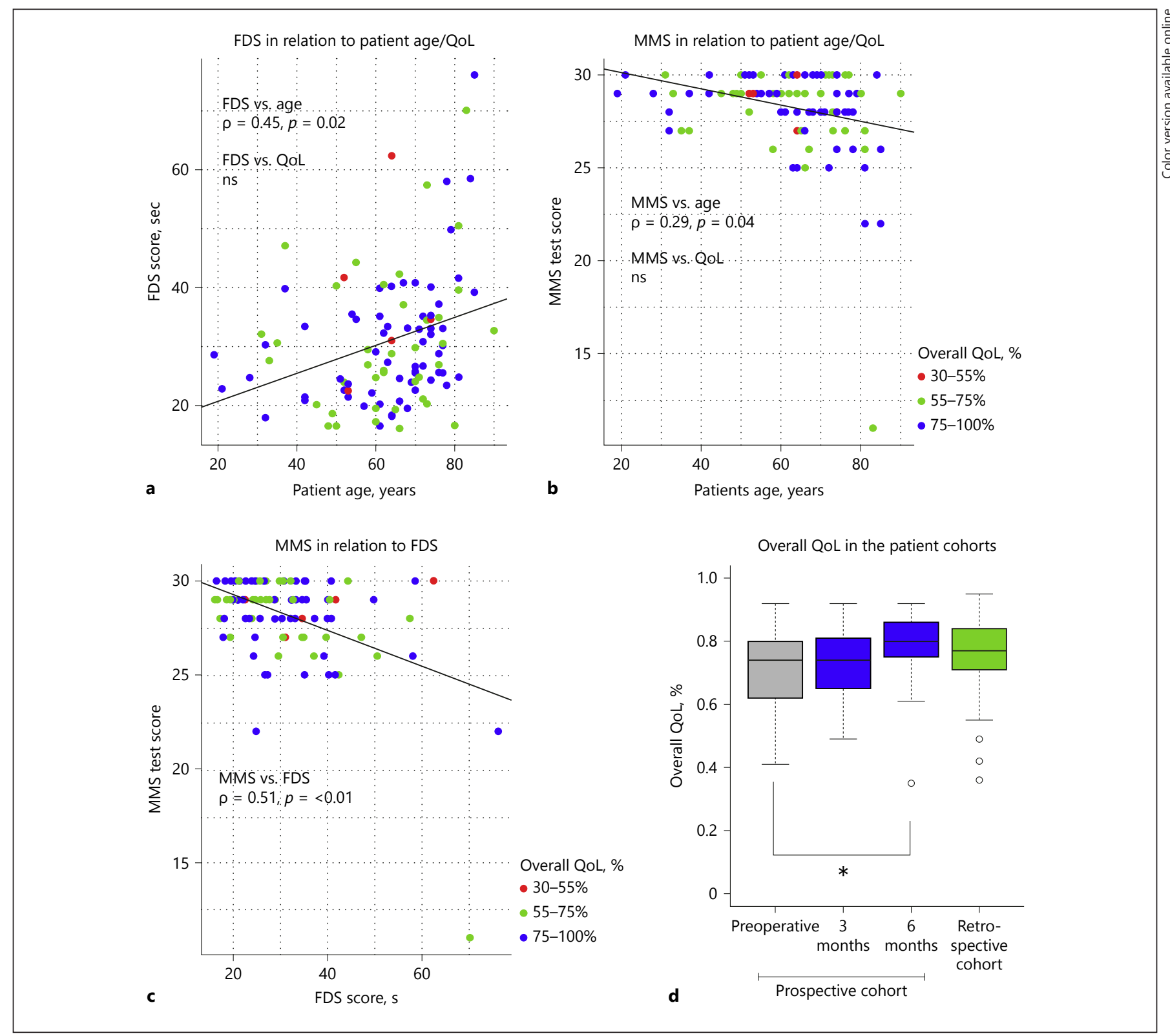

Fig. 1. General observations. a Dot plots showing the relation of FDS and patient age and HRQoL (colored dots) in the overall patient cohort. Pearson's $\rho$ (coeff.), the corresponding abline and the derived $p$ value is shown in the plots. $\mathbf{b}$ Dot plots showing the relation of MMS and patient age and HRQoL (colored dots) in the overall patient cohort. Pearson's $\rho$ (coeff.), the corresponding abline and the derived $p$ value is shown in the plot. $\mathbf{c}$ Dot plot show-

An Attempt of Simplification: The Clock Drawing Test In addition to FDS and MMS assessment, all participating patients also completed the clock drawing test, which is performed and interpreted quicker than the MMS test. Here, in accordance to the previously retrieved

Cognitive Status and Functional Dexterity ing the relation of MMS and FDS in the overall patient cohort. Pearson's $\rho$ (coeff.), the corresponding abline and the derived $p$ value is shown in the plot. $\mathbf{d}$ Boxplots showing the overall HRQoL in the prospective (grey and blue boxplots) and retrospective (green plot). $p$ values were calculated using the unpaired student's $t$ test. FDS, functional dexterity test; QoL, quality of life; MMS, mini mental status test; $\mathrm{ns}$, no significance. ${ }^{*} p$ values $<0.05$.

results, we found that patients with unimpaired clock drawing skills (score 1, median age 62) were significantly younger than patients with impaired clock drawing skills (score 2 and 3 , median age 72 and 74, respectively, $p \leq$ 0.01 ). Also, unimpaired clock drawing skills (score 1 , me- 


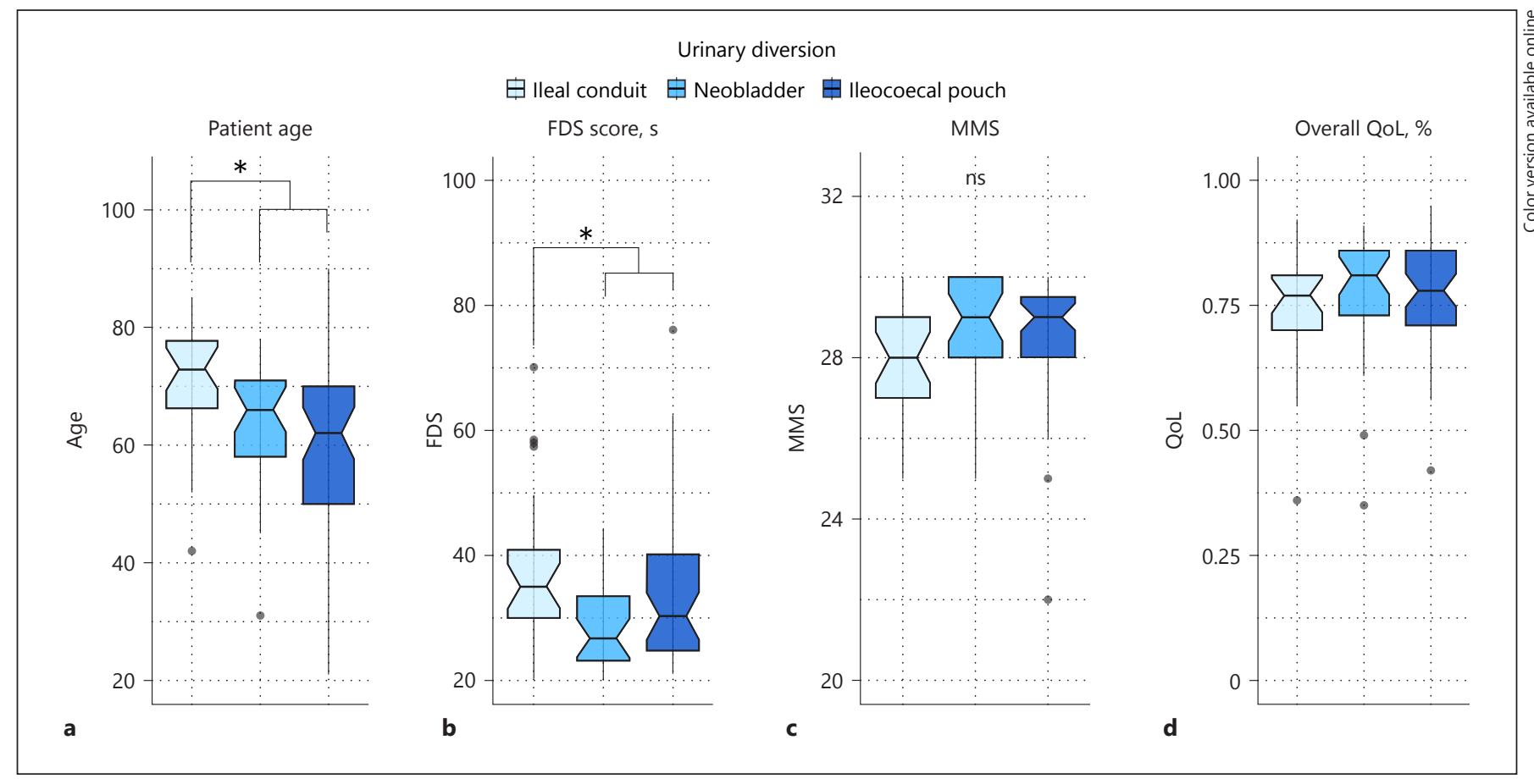

Fig. 2. Differences among the type of UD. Box plots showing the median (a) patients' age, (b) FDS scores, (c) MMS scores and (d) overall HRQoL percentage among the 3 different UDs (ileal conduit, neobladder, pouch). FDS, functional dexterity test; MMS, mental status test; QoL, quality of life; ns, no significance. ${ }^{*} p$ values $<0.05$.

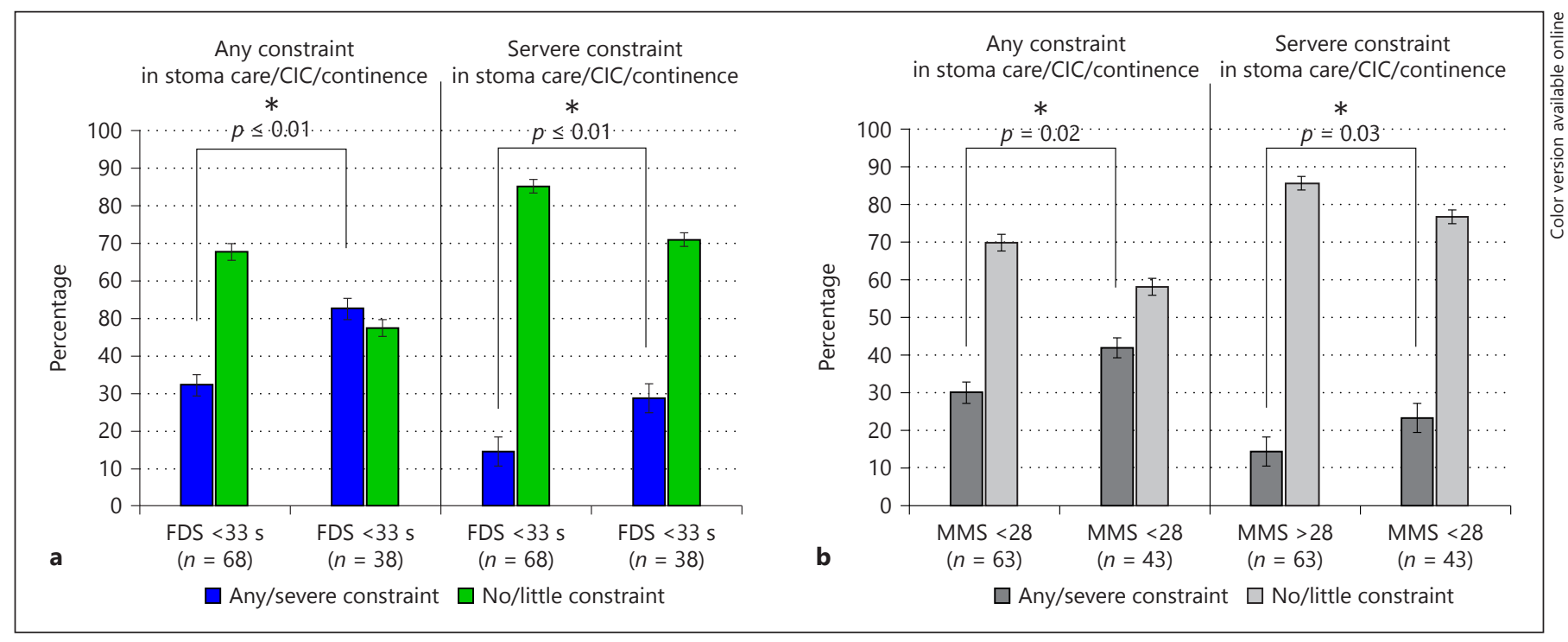

Fig. 3. Influence of FDS and MMS on constraints in stoma care/ $\mathrm{CIC/continence.} \mathrm{a} \mathrm{Bar} \mathrm{plots} \mathrm{showing} \mathrm{the} \mathrm{percentage} \mathrm{of} \mathrm{patients}$ with any (blue, left plots) or severe (blue bars, left plots) constraints regarding stoma care/CIC/continence in comparison to patients with little or no constraints (green bars), divided into patients with adequate (<33 s FDS) or impaired ( $>33$ s FDS) dexterity. b Bar plots showing the percentage of patients with any or severe (dark grey bars, left plots) constraints regarding stoma care/CIC/conti- nence in comparison to patients with little or no constraints (light grey bars), divided into patients with adequate (MMS $>28$ ) or impaired (MMS <28) cognitive performance. For definition of constraints, please refer to the third paragraph of the results part. All $p$ values were calculated using the chi-square test. CIC, self-catheterization; FDS, functional dexterity test; MMS, mental status test. $* p$ values $<0.05$. 


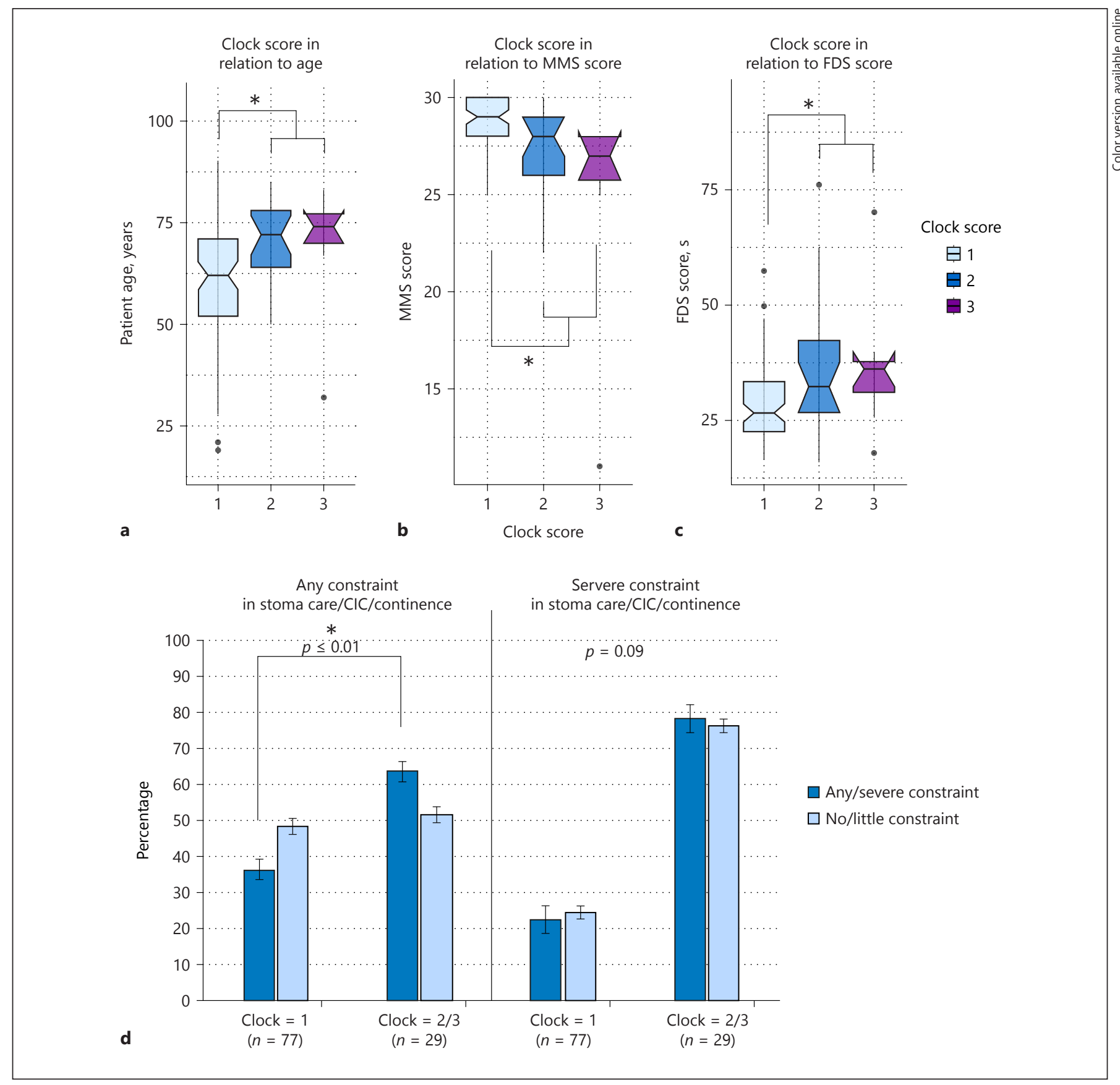

Fig. 4. Influence of the clock drawing test on age, MMS and FDS scores as well as on constraints in stoma care/CIC/continence. a-c Boxplots showing the relation of the clock score to patient age (a), MMS scores (b) and FDS scores (c). All $p$ values were calculated using the unpaired student's $t$ test. $\mathbf{d}$ Bar plots showing the percentage of patients with any or severe (dark blue bars, left plots) constraints regarding stoma care/CIC/continence in comparison to patients with little or no constraints (light blue bars), divided into patients with adequate $($ score $=1$ ) or impaired (score $=2$ or 3) clock drawing skills. For definition of constraints, please refer to the third paragraph of the results part. All $p$ values were calculated using the chi-square test. CIC, self-catheterization; FDS, functional dexterity test; MMS, mental status test. ${ }^{*} p$ values $<0.05$. 
dian MMS score 29) were significantly associated to higher MMS test scores than in patients with impaired clock drawing skills (score 2 and 3, median MMS scores 28 and $27, p \leq 0.01$ ). Concerning functional dexterity, a significantly progressive deterioration among the 3 different clock drawing scores was observed (score 1: $26.6 \mathrm{~s}$, score 2: $32.3 \mathrm{~s}$, score $3: 36.1 \mathrm{~s}, p \leq 0.01$ ) in forward analysis of variance. For functional outcomes, the clock drawing test was able to significantly discriminate patients with any or moderate constraints in stoma care, CIC, and continence (score 1: $36.4 \%$ constraints vs. score 2 and 3: $48.3 \%$ constraints, $p=0.01)$. However, for severe constraints, no statistical significance could be achieved (score 1: $22.1 \%$ constraints vs. score 2 and 3: $24.1 \%$ constraints, $p=0.63$ ). As for the MMS and FDS tests, overall HRQoL was not significantly affected by clock drawing skills (median $80 \%$ QoL measures among all groups, $p=0.1$ ). Figure 4 subsumes these results.

\section{Discussion}

An exhaustive number of studies have examined the surgical outcome including the issue of HRQoL after UD $[6-10,18,19]$; this study does not aim at re-inventing the wheel on this topic. In a nutshell, a comprehensive review by Gerharz et al. [18] revealed that there were no differences in long-term postoperative HRQoL among the different types of UD, be it a wet diversion or a continent solution. Due to lack of strong evidence (> level III) among the examined studies, the authors stated that prospective studies would be needed to further clarify this topic and answer remaining questions. For our research group, one of these questions was whether cognitive status or functional dexterity of the patients would impact functional outcomes, the medical care situation, or HRQoL after UD.

Our considerations about designing this study were steered by the increasing interest and demand concerning care research in urology (with special attention to the ageing society) $[11,20]$. Receiving an UD still remains a lifechanging experience; patients have to deal and live either with a "bag attached to the abdomen for urine storage" [18], CIC of an umbilical stoma, or voiding urine "via naturalis" over a hypocontractile spheroid created from intestine. Although the choice of UD apparently does not affect long-term HRQoL measures [18, 19], patients have to adapt to a certain medical care situation, be it stoma care, CIC, or voiding through a neobladder. In the latter cases, caring for oneself poses a greater mental and phys- ical challenge $[21,22]$ than, for example, frequent bag changes in an ileal conduit patient. Moreover, as mentioned before, the prospering occupational group of stoma therapists [12] can ease and cover most of patients' needs after creation of a wet loop UD compared to patients after a continent solution. Therefore, we hypothesized that better patient selection on the basis of objective, validated clinical tests would increase the subjective functional outcome and therefore the medical care situation in UD patients and may provide the treating clinician with a useful tool regarding which UD he or she recommends.

As objective tests assessing the mental and physical status of patients to receive a UD are not routinely established and sparse to no data are available on the topic to date, we chose the MMS/clock drawing test and the FDS test [13-16] for our study for 2 reasons. The first one is that all 3 tests are validated and in broad clinical use for over 20 years, giving them a certain credibility; the second one is that the FDS test somewhat resembles the act of catheterization and stoma care by demanding confident fine motor skills and one-handed dexterity from the patients $[13,16]$.

We found that reduced dexterity as well as poor cognitive function were significantly associated with patient age, which is confirmed by the existing literature on the topic $[14,23,24]$. As expected and mirrored by the existing literature, dexterity and cognitive function were also inversely correlated $[23,24]$. Interestingly, neither dexterity nor cognitive function seem to affect overall $\mathrm{HRQ}$ L in UD patients. We interpreted these results in the context that the term of HRQoL contains more than and is not dependent on manual dexterity and cognitive function, as other authors have stated and appropriately described as "the well-being paradoxon" before [18].

Based on these findings, we did a subgroup analysis of the 3 types of UDs examined in this study. We found that patients receiving an ileal conduit were significantly older and less dexterous than patients with a continent UD. No significances were found in cognitive function and overall HRQoL, confirming recent reviews on the topic $[7,18]$. In conjunction with the available literature, we conducted, that elderly patients usually bring along more comorbidities and therefore being offered "the easiest solution possible", the ileal conduit [2,25]. Based on these data and to further substantiate our claims that reduced dexterity or cognitive function would impair the medical care reality in the respective UDs, we found that impaired dexterity and cognitive function indeed was significantly associated with subjective constraints in stoma care, CIC, 
and continence. Therefore, we propose that these tests represent valid objective clinical tools to assess the medical care situation in patients with a UD. The well-disposed to reader might now wonder what manual dexterity has to do with continence after creation of a neobladder. Here, in our opinion, 2 aspects have to be considered: First, as mentioned before, voiding through a neobladder can pose a complex challenge upon the patient, both physically and mentally $[21,22]$. Taking this fact and the data we presented here into account, the additional information that dexterity correlates with cognitive function justifies the additional assessment of dexterity in patients eligible for an orthotopic continent UD. Secondly, hypercontinence in orthotopic continent UDs with the need for CIC in the follow-up period can occur in up to $10 \%$ of the patients [21], further strengthening the usefulness of dexterity assessment. We therefore propose further clinical evaluation of these tests in patients to receive a UD to validate our findings. In the light of the increasing work intensification at medical centers offering UD procedures, we know that time-consuming tests like the FDS and MMS test will most likely not find routine clinical use and propose that further simplification of the tests used is warranted. Therefore, we have also evaluated the clock drawing test [15], which is quickly performed, validated, and widely mirrors the results found for the time-consuming MMS and FDS tests. Time and further prospective evaluation will tell which of these tests will best meet the requirements both for the patients and for the treating clinicians. In this way, we propose that preoperative information of the patients regarding the respective UDs and the expected postoperative care situation will improve, therefore aiding in joint decision-making in the future.
Despite the noteworthy strengths of our study (e.g., the combined retrospective and prospective design as well as the comparably high number of patients with a continent cutaneous UD), limitations have to be mentioned: Due to the questionnaire-based design, well-known biases such as the examiner bias and sampling bias are to be considered. Moreover, the subjective assessment of constraints in UD medical care and the possible interference susceptibility of the FDS [16] and MMS [17] tests have to be interpreted within the limits of the used data collection.

Nonetheless, we think that we have opened the door towards an exciting new perspective in urological care research, which will hopefully encourage other researchers to deal with this topic in greater depth.

\section{Conclusions}

Our combined retrospective and prospective data imply that assessing the cognitive status and functional dexterity of patients undergoing UD might provide a useful objective preoperative tool to aid in decision-making regarding the type of UD and the postoperative medical care situation. However, further prospective data are needed to confirm these findings and further simplify the methods used here.

\section{Disclosure Statement and Ethics Statement}

None declared. All the findings, data acquisition, and processing in this study comply with the ethical standards laid down in the latest declaration of Helsinki as well as with the statutes of the Ethics Committee of the University of Würzburg concerning anonymized medical studies.

\section{References}

1 Tizzoni G, Foggi A: Die Wiederherstellung der Harnblase. Experimentelle Untersuchungen. Zentralbl Chir 1886;1:921.

2 Anderson CB, McKiernan JM: Surgical complications of urinary diversion. Urol Clin North Am 2018;45:79-90.

3 Goldwasser B, Webster GD: Continent urinary diversion. J Urol 1985;134:227-236.

4 Burkhard FC, Kessler TM, Mills R, Studer UE: Continent urinary diversion. Crit Rev Oncol Hematol 2006;57:255-264.

5 Wiesner C, Bonfig R, Stein R, Gerharz EW, PahernikS, Riedmiller H, ThüroffJW: Continent cutaneous urinary diversion: long-term follow-up of more than 800 patients with ileocecal reservoirs. World J Urol 2006;24:315-318.
6 Stein R, Rubenwolf P: Metabolic consequences after urinary diversion. Front Pediatr 2014; $2: 15$.

7 Leone A, Gilbert SM: Urinary diversion and health-related quality of life; in Urinary Diversion. Cham, Springer, 2017, pp 153169.

8 Cerruto MA, D’Elia C, Siracusano S, Saleh O, Gacci M, Cacciamani G, De Marco V, Porcaro $A B$, Balzarro $M$, Niero $M$, Lonardi $C$, Iafrate M, Bassi P, Imbimbo C, Racioppi M, Talamini R, Ciciliato S, Serni S, Carini M, Verze P, Artibani W: Health-related quality of life after radical cystectomy for bladder cancer in elderly patients with ileal orthotopic neobladder or ileal conduit: results from a multicentre cross-sectional study using validated questionnaires. Urol Int 2018;100:346352

9 Gierth M, Zeman F, Denzinger S, Vetterlein MW, Fisch M, Bastian PJ, Syring I, Ellinger J, Müller SC, Herrmann E, Gilfrich C, May M, Pycha A, Wagenlehner FM, Vallo S, Bartsch G, Haferkamp A, Grimm MO, Roigas J, Protzel C, Hakenberg OW, Fritsche HM, Burger M, Aziz A, Mayr R: Influence of body mass index on clinical outcome parameters, complication rate and survival after radical cystectomy: evidence from a prospective European multicentre study. Urol Int 2018;101:1624. 
10 Gschliesser T, Eredics K, Berger I, Szelinger $\mathrm{M}$, Klingler HC, Colombo T, Ponholzer A, Plas E, Grubmüller K, Dunzinger M, Jeschke K, Würnschimmel E, Krause FS, Shariat S, Leeb K, Pelzer A, Riedl C, Rauchenwald M, Hübner W, Brössner C, Madersbacher S; Cystectomy Registry of the Austrian Society of Urology: The impact of gender on tumour stage in In-House complications and choice of urinary diversion: results of the Austrian cystectomy registry. Urol Int 2017;99:429-435.

11 Biggs S: Adapting to an ageing society: the need for cultural change. Policy Quarterly 2014;10:12-16.

12 Bass EM, Del Pino A, Tan A, Pearl RK, Orsay $\mathrm{CP}$, Abcarian H: Does preoperative stoma marking and education by the enterostomal therapist affect outcome? Dis Colon Rectum 1997;40:440-442.

13 Aaron DH, Jansen CW: Development of the functional dexterity test (FDT): construction, validity, reliability, and normative data. J Hand Ther 2003;16:12-21.

14 Mitchell AJ: A meta-analysis of the accuracy of the mini-mental state examination in the detection of dementia and mild cognitive impairment. J Psychiatr Res 2009;43:411-431.
15 Mainland BJ, Shulman KI: Clock drawing test; in Cognitive Screening Instruments. London, Springer, 2013, pp 79-109.

16 Sartorio F, Bravini E, Vercelli S, Ferriero G Plebani G, Foti C, Franchignoni F: The functional dexterity test: test-retest reliability analysis and up-to date reference norms. J Hand Ther 2013;26:62-67.

17 Scazufca M, Almeida OP, Vallada HP, Tasse WA, Menezes PR: Limitations of the minimental state examination for screening dementia in a community with low socioeconomic status: results from the Sao Paulo Ageing \& Health Study. Eur Arch Psychiatry Clin Neurosci 2009;259:8-15.

18 Gerharz EW, Månsson $\mathrm{A}$, Hunt S, Skinner EC, Månsson W: Quality of life after cystectomy and urinary diversion: an evidence based analysis. J Urol 2005; 174:1729-1736.

19 Porter MP, Penson DF: Health related quality of life after radical cystectomy and urinary diversion for bladder cancer: a systematic review and critical analysis of the literature. J Urol 2005; 173:1318-1322.

20 Ghani KR, Zheng K, Wei JT, Friedman CP: Harnessing big data for health care and research: are urologists ready? Eur Urol 2014; 66:975-977.
21 Ginsberg DA, Rosenberg S: Neobladder voiding function in men. Curr Bladder Dysfunct Rep 2015;10:404-410.

22 Parekh DJ, Gilbert WB, Smith JA Jr: Functional lower urinary tract voiding outcomes after cystectomy and orthotopic neobladder. J Urol 2000;163:56-58; discussion 58-59.

23 Xie H, Zhang C, Wang Y, Huang S, Cui W, Yang W, Koski L, Xu X, Li Y, Zheng M, He M, Fu J, Shi X, Wang K, Tang G, Wang B, Huo Y: Distinct patterns of cognitive aging modified by education level and gender among adults with limited or no formal education: a normative study of the mini-mental state examination. J Alzheimers Dis 2016;49: 961-969.

24 de Paula JJ, Albuquerque MR, Lage GM, Bicalho MA, Romano-Silva MA, Malloy-Diniz LF: Impairment of fine motor dexterity in mild cognitive impairment and Alzheimer's disease dementia: association with activities of daily living. Rev Bras Psiquiatr 2016;38: 235-238.

25 Farnham SB, Cookson MS: Surgical complications of urinary diversion. World J Urol 2004;22:157-167. 